\title{
A-to-I editing sites are a genomically encoded G: Implications for the evolutionary significance and identification of novel editing sites
}

\author{
NAN TIAN, ${ }^{1}$ XIAOJIE WU, ${ }^{1}$ YAOZHOU ZHANG, ${ }^{2}$ and YONGFENG JIN ${ }^{1,2}$ \\ ${ }^{1}$ Institute of Biochemistry, College of Life Sciences, Zhejiang University, Hangzhou, Zhejiang, ZJ310058, People's Republic of China \\ ${ }^{2}$ Institute of Biochemistry, Zhejiang Sci-Tech University, Hangzhou, Zhejiang, ZJ 310018, People's Republic of China
}

\begin{abstract}
Ribonucleic acid (RNA) editing can extend transcriptomic and proteomic diversity by changing the identity of a particular codon. Genetic recoding as a result of adenosine-to-inosine (A-to-I) RNA editing can alter highly conserved or invariant coding positions in proteins. Interestingly, examples exist in which A-to-I editing sites in one species are fixed genomically as a $G$ in a closely related species. Phylogenetic analysis indicates that G-to-A mutations at the DNA level may be corrected by posttranscriptional A-to-I RNA editing, while in turn, the edited I (G) may be hardwired into the genome, resulting in an A-to-G mutation. We propose a model in which nuclear A-to-I RNA editing acts as an evolutionary intermediate of genetic variation. We not only provide information on the mechanism behind the evolutionary acquisition of an A-to-I RNA editing site but also demonstrate how to predict nuclear A-to-I editing sites by identifying positions where an RNA editing event would maintain the conservation of a protein relative to its homologs in other species. We identified a novel edited site in the fourth exon of the cacophony transcript coding calcium channel $\alpha 1$ and verified it experimentally.
\end{abstract}

Keywords: A-to-I RNA editing; G-to-A mutation; evolutionary significance; cacophony

\section{INTRODUCTION}

Ribonucleic acid (RNA) editing is a process resulting in the synthesis of proteins that are not directly encoded in the genome. One type of RNA editing involves the modification of individual adenosine (A) bases to inosine (I) in RNA by adenosine deaminases acting on RNA (ADAR) enzymes (Bass 2002; Maas et al. 2003). Since I acts as guanosine (G) during translation, A-to-I conversion in coding sequences leads to amino acid changes and often entails changes in protein function (Seeburg et al. 1998; Bass 2002; Schmauss and Howe 2002). A-to-I RNA editing is common in animals and is associated with various neurological functions (Seeburg et al. 1998; Schmauss and Howe 2002). Caenorhabditis elegans, Drosophila, and mouse mutants lacking ADAR enzymes have distinct neurological phenotypes (Higuchi et al. 2000; Palladino et al. 2000; Tonkin et al.

Reprint requests to: Yongfeng Jin, Institute of Biochemistry, College of Life Sciences, Zhejiang University (Zijingang Campus), Hangzhou, Zhejiang, ZJ310058, People's Republic of China; or Institute of Biochemistry, Zhejiang Sci-Tech University, Hangzhou, Zhejiang, ZJ 310018, People's Republic of China; e-mail: jinyf@zju.edu.cn; fax: +86-571-88206478.

Article published online ahead of print. Article and publication date are at http://www.rnajournal.org/cgi/doi/10.1261/rna.797108.
2002; Wang et al. 2000). In addition to amino acid changes, the editing and subsequent destabilization of the RNA duplex present in the $5^{\prime}$ - or $3^{\prime}$-untranslated regions (UTRs) could alter the stability, transport, or translation of the mRNA (Knight and Bass 2001; Tonkin and Bass 2003). Moreover, RNA editing could influence alternative splicing decisions (Rueter et al. 1999; Schoft et al. 2007), antagonize RNA interference (RNAi), and redirect microRNA (miRNA) silencing targets (Knight and Bass 2002; Tonkin and Bass 2003; Kawahara et al. 2007).

In animals, genetic recoding by A-to-I RNA editing is most common, altering highly conserved or invariant coding positions in proteins (Reenan 2005). However, some nuclear A-to-I editing sites are shown to be genomically encoded as a $\mathrm{G}$ in other species (Grauso et al. 2002; Jin et al. 2007; Ohlson et al. 2007). It is not clear whether this represents the ancestral state, or, whether high levels of editing of an ancestor were "hardwired" into the genome through missense mutation. In this paper, we show that at least some A-to-I RNA editing functions to correct G-to-A mutations, while in turn, the edited I (G) was hardwired into the genome. Based on this model, we predicted a novel A-to-I editing site and verified it experimentally. 


\section{RESULTS AND DISCUSSION}

\section{A-to-I editing sites as compensators of G-to-A mutations}

In animals, A-to-I RNA editing usually alters highly conserved or invariant coding positions in proteins (Reenan 2005). Interestingly, several examples exist in which an A-to-I editing site represents a genomically encoded $\mathrm{G}$ in a related species. For example, site 15 in nAChR $\alpha 6$ is edited in Bombyx mori and Apis mellifera, while the corresponding site of the $\alpha 6$ ortholog $\alpha 7-2$ in the tobacco budworm Heliothis virescens (Lepidoptera) harbors a genomically encoded G (Fig. 1A). Site 3 is edited in A. mellifera, while the homologous sites in Anopheles gambiae, H. virescens, B. mori, and Tribolium castaneum consist of a genomically encoded G (Fig. 1A). Similarly, the $\alpha 6$ site 2 is edited in Drosophila (Grauso et al. 2002) and Musca domestica
(Deacutis et al. 2007), while the homologous $\alpha 6$ sites in other species consist of genomically encoded Gs (Fig. 1A). Two editing sites (Fig. 1A, sites 5,12) in nAChR $\alpha 6$ are conserved in four orders of insect, represented by Drosophila melanogaster, B. mori, T. castaneum, and A. mellifera. This is considered the most highly conserved RNA editing event yet reported in invertebrates. These two homologous $\alpha 6$ sites have a genomically encoded G in Pediculus humanus (Phthiraptera) (Fig. 1A). The situation is similar for the I/M editing site of GABAA receptor subunit $\alpha 3$, where the frog and pufferfish have a genomically encoded methionine at the equivalent position, while more evolved species have an I codon in the same position (Ohlson et al. 2007). The situation is also similar for the $Q / R$ editing site of the glutamate receptor subunit gene B (GluR-B); the hagfish, a jawless fish, and teleosts have an $\mathrm{R}$ codon at this position, while species that appeared after the cartilaginous fish have a $\mathrm{Q}$ codon in the same position (Wu et al. 1996; Kung et al. 2001). Although we do not know the generality of this phenomenon, it led us to determine whether the genomically encoded $G$ represents the ancestral state, or whether high levels of editing of an ancestor were hardwired into the genome through missense mutations.

\section{G-to-A mutations may be corrected by A-to-I RNA editing}

The evolutionary history of the editing of nAChR $\alpha 6$ is charted roughly in insects. Figure 1 shows that site 2 is edited in the nAChR $\alpha 6$ of D. melanogaster and $M$. domestica, while the homologous sites in A. gambiae, $H$. virescens, $T$. castaneum, B. mori, and $P$. humanus have a genomically encoded G. Correspondingly, these orthologs encode $\mathrm{V}$ at the position of this $\mathrm{I} / \mathrm{V}$ editing site. Phylogenetic analysis indicates that $G$ represents the ancestral state at this site, at least before the radiation of the Drosophilidae and Culicidae (spanning $\sim 200$ million years). We also analyzed these orthologs from 12 Drosophila species (D. melanogaster, $D$. simulans, D. sechellia, D. yakuba, D. erecta, D. ananassae, D. pseudoobscura, D. persimilis, D. willistoni, D. mojavensis, D. virilis, and D. grimshawi) and found that the corresponding position encodes an $\mathrm{A}$ in these orthologs. This indicates that G-to-A mutations 
occurred at least before the radiation of the Drosophilidae and Culicidae.

Similarly, site 8 is edited in $M$. domestica, while the position of this editing site in D. melanogaster, A. gambiae, $H$. virescens, $T$. castaneum, $B$. mori, and $P$. humanus contains a genomically encoded G (Fig. 1A). Again, phylogenetic analysis indicates that a $G$ represents the ancestral state at this site, at least before the radiation of the Drosophilidae and Culicidae. At the divergence of the Muscidae and Drosophilidae, the G was converted into an A in the Muscidae, while a G remained in drosophilids. Analysis of these orthologs from 12 Drosophila species ( $D$. melanogaster, D. simulans, D. sechellia, D. yakuba, D. erecta, D. ananassae, D. pseudoobscura, D. persimilis, D. willistoni, D. mojavensis, $D$. virilis and $D$. grimshawi) showed that they possess a G. Compared with site 2, the conversion at site 8 is confined to a smaller phyletic branch.

Although the G-to-A mutation probably occurred against negative selective pressure, subsequent A-to-I RNA editing counteracted this conversion, maintaining similarity at the protein level to reduce negative selective pressure. Therefore, the A-to-I RNA editing of this site must have evolved immediately after the G-to-A conversion event. Consistently, the converted A was located within a secondary structure. Subsequently, RNA editing at this position may have evolved to be dispensable and has consequently been abolished due to loss of the corresponding secondary structure, such as in A. gambiae (Fig. 1A). Unlike other A-to-I editing sites, these editing sites represent an atypical A-to-I editing event, which increases the conservation of a protein relative to homologs in other species.

However, this occurrence of A-to-I RNA editing cannot be fully explained in terms of correcting G-to-A mutations. Mutational data show that the AMPA receptor subunit of GluR-B in its unedited Q/R site form is not essential for brain development and function (Kask et al. 1998); however, this is the only editing site with nearly $100 \%$ frequency throughout development in rodents. Unlike the case of GluR-B, most genes are partially edited or edited in a tissue-dependent manner. This implies that a more complex editing mechanism has evolved in the nuclear genome, one that plays a role in regulating gene expression, in addition to neutralizing G-to-A mutations. The evolutionary conservation of the spatial and developmental regulation of RNA editing also suggests that the extent of editing may modulate biological function. Much evidence indicates that the unedited RNA was important biologically (Vissel et al. 2001). Moreover, an early gene might be edited at an optimal, yet intermediate, level. Over time, the subsequent mutations in double-stranded RNA (dsRNA) regions may have allowed higher levels of editing, even 100\% editing, to achieve the maximum compensatory level. However, such $100 \%$ editing is rarely found at all developmental stages, and perhaps partial RNA editing may be sufficient to correct G-to-A mutations.

\section{Edited nucleotides may be hardwired into the genome}

Site 15 is edited in the $\mathrm{nAChR} \alpha 6$ of $B$. mori and $A$. mellifera, while this site in the $\alpha 6$ ortholog $\alpha 7-2$ in the tobacco budworm $H$. virescens has a genomically encoded G (Fig. 1A). Phylogenetic analysis indicates that A represents the ancestral state at this site, which gained A-to-I RNA editing during evolution. A-to-G conversion tends to occur because of the low negative selective pressure at the protein and functional levels; consequently, edited nucleotides may be hardwired into the genome. This hypothesis appears reasonable because eliminating the requirement for editing by engineering a constitutive $\mathrm{R}$ at the GluR-B gene Q/R site had no obvious effects in mice (Kask et al. 1998) and was able to rescue the lethal phenotype observed in ADAR2 knockout mice (Higuchi et al. 2000).

\section{The implications of A-to-I RNA editing as an evolutionary intermediate of genetic variation}

Based on the comparative data presented above, we proposed a model for the evolutionary mechanism of A-to-I RNA editing (Fig. 2). The first consideration of this model is that $\mathrm{G}$ represents the ancestral state and was converted into A during evolution. A-to-I RNA editing occurred immediately after the G-to-A conversion event, maintaining similarity at the protein level to reduce negative selective pressure (Fig. 2A). In this case, the G-to-A mutation at the DNA level may be corrected by post-transcriptional A-to-I RNA editing. The second consideration of this model is that A represented the ancestral state, which gained A-to-I RNA editing during evolution. A-to-G conversion tended to occur because of low negative selective pressure, so that edited nucleotides might be hardwired
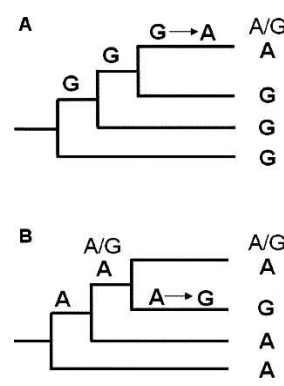

FIGURE 2. Model of the evolutionary mechanism of nuclear A-to-I RNA editing as an evolutionary intermediate of genetic variation. $(A)$ $G$ represents the ancestral state, which was converted to A during evolution. A-to-I RNA editing occurred immediately after the G-to-A conversion event, maintaining similarity at the protein level to reduce negative selective pressure. A-to-I RNA editing might be lost in the subsequent evolutionary step. $(B)$ A represents the ancestral state, which gained A-to-I RNA editing during evolution. A-to-G conversion tended to occur because of low negative selective pressure, and thus the edited nucleotides might be hardwired into the genome. $\mathrm{G} \rightarrow \mathrm{A}$ and $\mathrm{A} \rightarrow \mathrm{G}$ denote $\mathrm{G}-$ to-A and A-to-G mutations, respectively, and A/G indicates A-to-I RNA editing. 
into the genome, resulting in an A-to-G mutation at the DNA level (Fig. 2B). In any case, nuclear A-to-I RNA editing might act as an evolutionary intermediate of A-to-G or G-to-A mutation, maintaining conservation at the protein and functional levels during evolution despite sequence divergence at the DNA level.

Does RNA editing affect genetic variation? Comparative analysis indicated that the fifth exon of the nAChR $\alpha 6$ encodes 61 amino acids, of which 50 (85\%) are conserved in all eight species. Sixteen edited sites exist, resulting in 12 amino acid changes, of which only five of 12 (42\%) are conserved in all eight species. This suggests that the edited sites accumulate mutations nearly twice as rapidly as the unedited sites. Moreover, the prevalence of $\mathrm{A} / \mathrm{G}$ and $\mathrm{C} / \mathrm{T}(\mathrm{U})$ substitutions in the genetic variation might be explained by the fact that the most prevalent changes of RNA editing in the nuclei of higher eukaryotes are hydrolytic deaminations, in which the genomically encoded $\mathrm{C}$ or $\mathrm{A}$ is converted into $\mathrm{U}$ and $\mathrm{I}$, respectively (Brennicke et al. 1999). Therefore, nuclear A-to-I RNA editing not only extends the sequence diversity at the RNA and protein levels but is also a novel source of genetic variation, at least in some genome sequences.

\section{Detection and verification of a novel A-to-I editing site}

Since nuclear A-to-I RNA editing tends to increase protein conservation across species by "correcting" codons that specify less-conserved amino acids in some cases, this principle can be used to predict A-to-I editing sites by identifying positions where an RNA editing event would increase the conservation of a protein relative to homologs from other species. One of the most promising predicted structures suitable for A-to-I editing was found in the fourth exon of the cacophony (cac) transcript coding the calcium channel $\alpha 1$ subunit. A-to-I editing has been identified at 12 positions (Smith et al. 1996; Keegan et al. 2005), while A-to-I editing sites in the fourth exon have not been studied. This region mainly encodes transmembrane segment S4 and the preceding extracellular loop in the first repeat domain of this channel (Fig. 3A). Phylogenetic analysis indicated that $\mathrm{G}$ represented the ancestral state at this site. Until the radiation of the Sophophora and Drosophila groups (spanning $\sim 41$ million years), $G$ was mutated to A at this site in the Drosophila groups. Therefore, the I/M sites of cac in D. mojavensis, D. virilis, and D. grimshawi have a genomically encoded $M$, while the species that appeared after the Drosophila group have an I at the same position. The homologous site is also a genomically

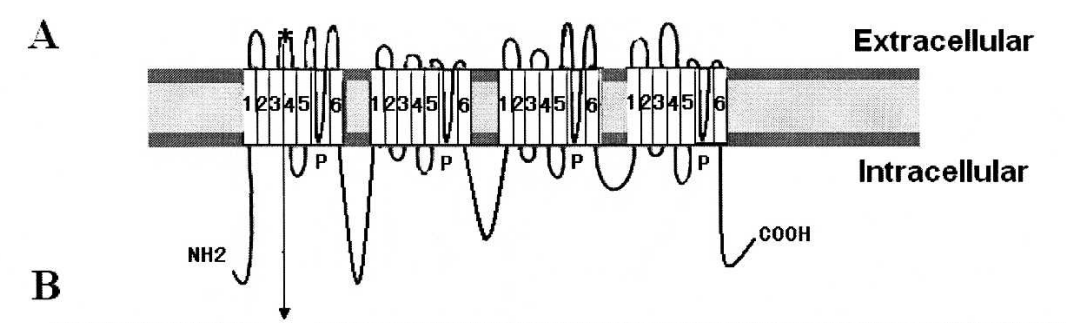

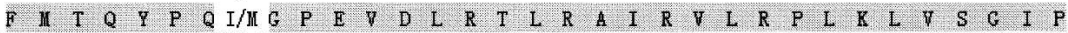
DMe TTCATGACACACTIOCCACAAATAGGGCOCGAGTTAGACCTAAGAACACTTAGAGCCATTOGTGTGCTACGGCCCTTAAAATTAGTETCTGGAATTCCT Ds i TTCATGACACACTACCCACAAATAGGGCOCGAGGTHGACETAAGAACACTTAGGGCCATTOGTGTECTACGGCCCTTAAA ATTAGTGTCTGGAATTCCT DSE TTCATGACACACTACCCACAAATAGGOCOCCAGGTACGOCTAACAACACTTAGACCCATTOGTGTGCTACCGCCCTTAAAATTACTGTCTGCAATTCGT DYO TTCATGACACAGTACCCACAAATAGGCCOOGAGGTAGAOCTABGAACACTTAGAGCCA TTOGTETGCTACGGOCETTAAAATTAGTGTCTGEAATTCCT Der TTCATGACACACTICCCACAALTAGGCCOCGAGGTAGACTTAGAACACTTAGACCCATTCGTETECTACGCOCCTTALATTAGTETCTGGAATTCCT Dan TTCATGACACAGTAOCCACAAATAGEGCOCGAGGTAGAOCTAAGAACACTTAGAGCCATTOGTGTGCTACGGCCOCTAAAATTAGTGTCTGCAATTCCT Dps TTCATGACACAGTAOCCACAATTACGGCOCGAGGTAGAOCTAAGAACACTTAGAGCCATTOCTGTGCTACGGOCCTTAAAATTAGTGTCTGGAATTCCT Dpe TTCATGACACAGTACCCACAAATAGGGCOCGAGGTACAOUTARGABCACTTAGACCCATTOGTGTGCTACGCOCGTTAAA BTTAGTGTCTGGABTTCGT

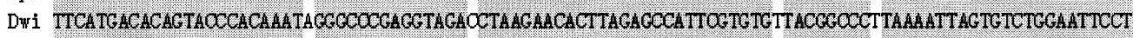
DMO TTCATGACACAGTACCCACAAATGCGCCOCGACCTAGATCTAACAACACTTAGACCCATTOGTGTGCTACGGCCCTTAAAATTEGTGTCTGGAATTCCT

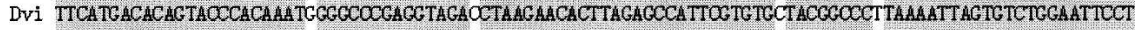
Dgr TTCATGACACAGTACCCACAA TGGGGCOCGAGGTAGAOCTAAGAACACTTAGAGCCATTOGTGTECTACGGOCCTTAAAATTAGTGTCTGGAATTCCT

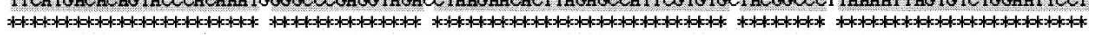

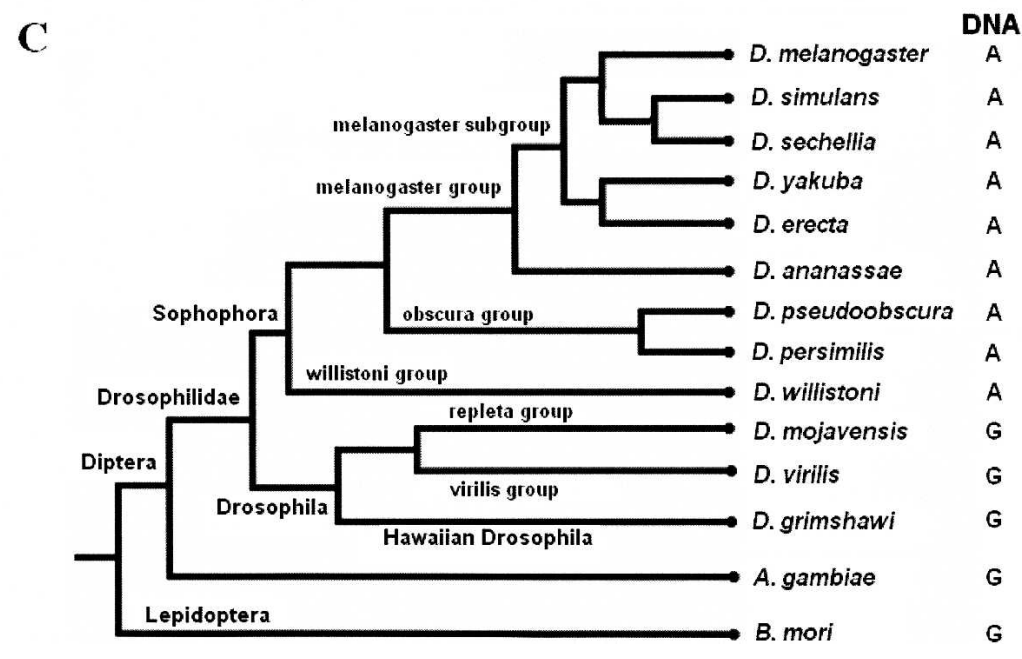

FIGURE 3. Position of the edited site in cac and the evolutionary analysis. $(A)$ The protein sequence of cac consists of four repeating transmembrane domains (TM1-TM4), each including six transmembrane segments (S1-S6) (Catterall 2000; Keegan et al. 2005). Shading represents the plasma membrane. Both the $\mathrm{N}$ and $\mathrm{C}$ termini of the $\alpha 1$ subunit are intracellular, and the four repeats are linked by three major intracellular loops. The edited site (shown by the " *») was located within the extracellular loop between transmembrane segments S3 and S4 of the first repeating domain. (B) The I/M sites of cac in D. mojavensis (Dmo), D. virilis (Dvi), and D. grimshawi (Dgr) have a genomically encoded methionine, while D. melanogaster (Dme), D. simulans (Dsi), D. sechellia (Dse), D. yakuba (Dya), D. erecta (Der), D. ananassae (Dan), D. pseudoobscura (Dps), D. persimilis (Dpe), and D. willistoni (Dwi) have an isoleucine. Shading represents conserved nucleotide and amino acid sequences across Drosophila species. $(C)$ Evolutionary tree showing the phylogenetic relationships among 12 Drosophila species, one mosquito species (A. gambiae), and the silkworm (B. mori). 
encoded G in A. gambiae and B. mori. The situation is similar for sites 2 and 5 of nAChR $\alpha 6$ (Fig. 1A), although this conservation is confined to a younger phyletic branch. Therefore, we predicted that A-to-I editing occurred at this site. In parallel, we predicted a weak stem-loop structure suitable for A-to-I editing in the encoded sequences using the program mFold (Zuker 2003).

To validate the predicted editing site of the calcium channel $\alpha 1$ gene experimentally, we isolated total RNA from an adult $D$. melanogaster and compared the sequence determined after RT-PCR with the genomic sequence. An adenosine was present in the genomic sequence of exon 4 at the position in which a $\mathrm{G}$ and A mixed signal was present in the cDNA sequence (Fig. 4). At this position, an isoleucine (I) AUA codon is changed to an AUI and read as a methionine (M) codon (AUG), since inosine is read as a guanosine. RNA editing of the I/M site was confirmed for the other Drosophila species: D. simulans, D. sechellia, D. yakuba, D. erecta, and D. ananassae. To investigate if editing at the I/M site of cac changes during development, total RNA was extracted from fruit fly embryos, larvae, pupae, and adults. The extent of editing was determined by sequencing individual clones derived from the RT-PCR products. As a result, the $\mathrm{I} / \mathrm{M}$-site in the cacophony tran-

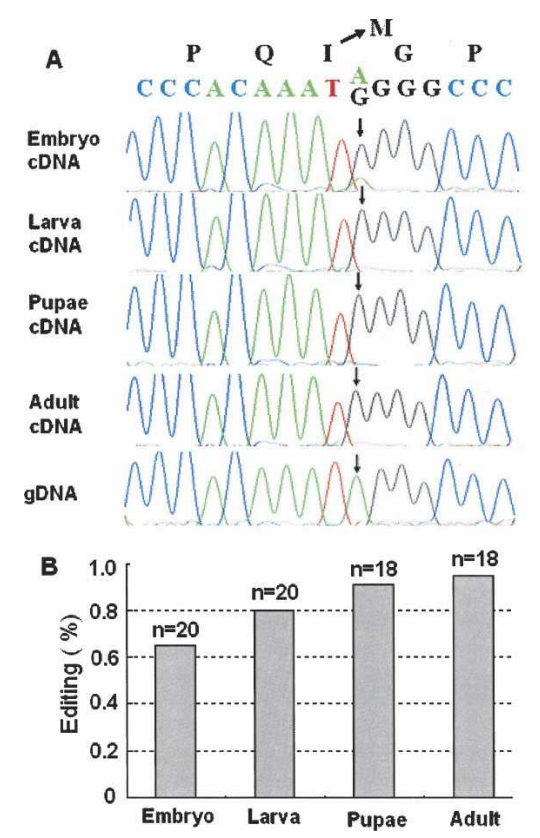

FIGURE 4. A-to-I editing site of the calcium channel $\alpha 1$ gene. $(A)$ Reverse transcriptase reactions were performed with oligo $(\mathrm{dT})_{15}$ on poly(A)+ RNA from D. melanogaster embryos, larvae, pupae, and adults. The RT-PCR and PCR products corresponding to the mRNAs were purified for direct sequencing. The editing sites (arrow) show a mixed $A / G$ or pure $G$ signal, while the DNA sequence contains adenosine signals only. $(B)$ RT-PCR products from the embryos, larvae, pupae, and adults were cloned, and relative editing levels were analyzed by sequencing. The number $(n)$ of clones analyzed for each sample is indicated. script shows an increase in RNA editing through development. This trend is similar to that at other editing sites and is consistent with increasing levels of ADAR expression through development, which is most evident in adult flies (Keegan et al. 2005). Since we have insufficient editing data, we cannot determine the generality of this rule; however, its importance lies in the fact that the resulting editing occurs in the coding region of a gene. We will be able to make broader generalizations, particularly for these atypical editing sites, after considering the other main criteria regarding editing probability, such as dsRNA regions.

\section{CONCLUSIONS}

In conclusion, we found several atypical nuclear editing events that generated a conserved codon identity at the RNA level, but not at the DNA level. G-to-A mutations at the DNA level may be corrected by post-transcriptional A-to-I RNA editing, while in turn, the edited I (G) may also be hardwired into the genome, resulting in an A-to-G mutation. Therefore, nuclear A-to-I RNA editing might act as an evolutionary intermediate of genetic variation. We proposed a model for the evolutionary mechanism of nuclear A-to-I RNA editing, and based on this model, we identified a novel edited site in the fourth exon of the cacophony transcript coding the calcium channel $\alpha 1$ and verified it experimentally.

\section{MATERIALS AND METHODS}

\section{Materials}

Total RNA was isolated from D. melanogaster at different developmental stages using the RNeasy Mini Kit (Qiagen) according to the manufacturer's protocol. Genomic DNA was isolated using the Universal Genomic DNA Extraction Kit (TaKaRa). Plasmid DNA was purified using a Qiagen plasmid isolation kit.

\section{Gene analysis}

The sequences of the nAChR subunit $\alpha 6$ genes from $D$. melanogaster, M. domestica, $H$. virescens, B. mori, A. gambiae, $T$. castaneum, and $A$. mellifera have been described previously (Grauso et al. 2002; Jones et al. 2005; Deacutis et al. 2007; Jin et al. 2007). The sequences of the nAChR subunit $\alpha 6$ genes from the human body louse, $P$. humanus, were assembled from individual raw sequence reads available from the NCBI trace archives. The sequences of the cac genes for the other Drosophila species (D. sechellia, D. yakuba, D. erecta, D. ananassae, D. pseudoobscura, D. persimilis, D. willistoni, D. mojavensis, D. virilis, and D. grimshawi), A. gambiae, and B. mori were assembled from individual raw sequence reads available from the NCBI trace archives. The sequence of the cac gene for D. simulans was determined using PCR and sequencing. The nucleotide and amino acid sequences of each alternative exon from each species were aligned using the program ClustalW (EMBL-EBI). 


\section{Analysis of RNA editing}

RNA editing was analyzed using total RNA from D. melanogaster as the template for RT-PCR, which was performed using primer pairs for the cac gene in different species. The primers used to amplify the RT-PCR products were 5'-GTGTAGAAGCGTCGCT CAAGATCC-3' (forward) and 5'-ACTAGGAATTCCAGACACT-3' (reverse). The PCR products were sequenced directly after gel purification. Primers for the cac gene exon 4 used to amplify genomic DNA from the same tissues used for RNA isolation were 5'-CCATAGTTTACATTTGCTCT-3' (forward) and 5' -ACTAGG AATTCCAGACACT-3' (reverse). The genomic PCR amplification product was sequenced directly to demonstrate that the genomic products give a pure A signal at editing sites, ruling out a polymorphism. In addition, the RT-PCR products were cloned into the pGEM-T Easy vector (Promega), and $\sim 20$ cDNA clones were sequenced for every stage to determine the relative A-to-G abundance. RNA editing of the I/M site was confirmed using RT-PCR analysis from adult total RNA and direct sequencing analysis for the other Drosophila species: D. simulans, D. sechellia, D. yakuba, D. erecta, and D. ananassae.

\section{ACKNOWLEDGMENTS}

We acknowledge Michael Jantsch for help in the writing of the manuscript. This work was partly supported by research grants from the National Natural Science Foundation of China (90508007 and 30770469), the 863 Program (2006AA10A119), and the Program for New Century Excellent Talents in University (NCET-04-0531).

Received August 27, 2007; accepted October 31, 2007.

\section{REFERENCES}

Bass, B.L. 2002. RNA editing by adenosine deaminases that act on RNA. Annu. Rev. Biochem. 71: 817-846.

Brennicke, A., Marchfelder, A., and Binder, S. 1999. RNA editing. FEMS Microbiol. Rev. 23: 297-316.

Catterall, W.A. 2000. Structure and regulation of voltage-gated $\mathrm{Ca}^{2+}$ channels. Annu. Rev. Cell Dev. Biol. 16: 521-555.

Deacutis, J.M., Scott, J.G., and Gao, J.R. 2007. The nicotinic acetylcholine receptor subunit Md $\alpha 6$ from Musca domestica is diversified via post-transcriptional modification. Insect Mol. Biol. 16: $325-334$.

Grauso, M., Reenan, R.A., Culetto, E., and Sattelle, D.B. 2002. Novel putative nicotinic acetylcholine receptor subunit genes, D $\alpha 5, \mathrm{D} \alpha 6$, and $\mathrm{D} \alpha 7$, in Drosophila melanogaster identify a new and highly conserved target of adenosine deaminase acting on RNA-mediated A-to-I pre-mRNA editing. Genetics 160: 1519-1533.

Higuchi, M., Maas, S., Single, F.N., Hartner, J., Rozov, A., Burnashev, N., Feldmeyer, D., Sprengel, R., and Seeburg, P.H. 2000. Point mutation in an AMPA receptor gene rescues lethality in mice deficient in the RNA-editing enzyme ADAR2. Nature 406: 78-81.

Jin, Y.F., Tian, N., Cao, J., Liang, J., Yang, Z., and Lv, J. 2007. RNAediting and alternative splicing of the insect nicotinic acetylcholine receptor subunit $\alpha 6$ transcript: Evolutionary conservation, divergence, and regulation. BMC Evol. Biol. 7: 98.

Jones, A.K., Grauso, M., and Sattelle, D.B. 2005. The nicotinic acetylcholine receptor gene family of the malaria mosquito, Anopheles gambiae. Genomics 85: 176-187.
Jones, A.K., Raymond, D.V., Thany, S.H., Gauthier, M., and Sattelle, D.B. 2006. The nicotinic acetylcholine receptor gene family of the honeybee, Apis mellifera. Genome Res. 16: 1422-1430.

Kask, K., Zamanillo, D., Rozov, A., Burnashev, N., Sprengel, R., and Seeburg, P.H. 1998. The AMPA receptor subunit GluR-B in its Q/ $\mathrm{R}$ site-unedited form is not essential for brain development and function. Proc. Natl. Acad. Sci. 95: 13777-13782.

Kawahara, Y., Zinshteyn, B., Sethupathy, P., Iizasa, H., Hatzigeorgiou, A.G., and Nishikura, K. 2007. Redirection of silencing targets by adenosine-to-inosine editing of miRNAs. Science 315: 1137-1140.

Keegan, L.P., Brindle, J., Gallo, A., Leroy, A., Reenan, R.A., and O'Connell, M.A. 2005. Tuning of RNA editing by ADAR is required in Drosophila. EMBO J. 24: 2183-2193.

Knight, S.W. and Bass, B.L. 2001. A role for the RNase III enzyme DCR-1 in RNA interference and germ line development in Caenorhabditis elegans. Science 293: 2269-2271.

Knight, S.W. and Bass, B.L. 2002. The role of RNA editing by ADARs in RNAi. Mol. Cell 10: 809-817.

Kung, S.S., Chen, Y.C., Lin, W.H., Chen, C.C., and Chow, W.Y. 2001. Q/R RNA editing of the AMPA receptor subunit 2 (GRIA2) transcript evolves no later than the appearance of cartilaginous fishes. FEBS Lett. 509: 277-281.

Maas, S., Rich, A., and Nishikura, K. 2003. A-to-I RNA editing: Recent news and residual mysteries. J. Biol. Chem. 278: 1391-1394.

Ohlson, J., Pedersen, J.S., Haussler, D., and Öhman, M. 2007. Editing modifies the GABAA receptor subunit $\alpha 3$. RNA 13: 698-703.

Palladino, M.J., Keegan, L.P., O'Connell, M.A., and Reenan, R.A. 2000. A-to-I pre-mRNA editing in Drosophila is primarily involved in adult nervous system function and integrity. Cell 102: 437-449.

Reenan, R.A. 2005. Molecular determinants and guided evolution of species-specific RNA editing. Nature 434: 409-413.

Rueter, S.M., Dawson, T.R., and Emeson, R.B. 1999. Regulation of alternative splicing by RNA editing. Nature 399: 75-80.

Schmauss, C. and Howe, J.R. 2002. RNA editing of neurotransmitter receptors in the mammalian brain. Sci. STKE 133: pe26. doi: 10.1126/stke.2002.133.pe26.

Schoft, V.K., Schopoff, S., and Jantsch, M.F. 2007. Regulation of glutamate receptor B pre-mRNA splicing by RNA editing. Nucleic Acids Res. 35: 3723-3732. doi: 10.1093/nar/gkm314.

Seeburg, P.H., Higuchi, M., and Sprengel, R. 1998. RNA editing of brain glutamate receptor channels: Mechanism and physiology. Brain Res. Rev. 26: 217-229.

Smith, L.A., Wang, X.J., Peixoto, A.A., Neumann, E.K., Hall, L.M., and Hall, J.C. 1996. A Drosophila calcium channel al subunit gene maps to a genetic locus associated with behavioural and visual defects. J. Neurosci. 16: 7868-7879.

Tonkin, L.A. and Bass, B.L. 2003. Mutations in RNAi rescue aberrant chemotaxis of ADAR mutants. Science 302: 1725.

Tonkin, L.A., Saccomanno, L., Morse, D.P., Brodigan, T., Krause, M., and Bass, B.L. 2002. RNA editing by ADARs is important for normal behavior in Caenorhabditis elegans. EMBO J. 21: 60256035.

Vissel, B., Royle, G.A., Christie, B.R., Schiffer, H.H., Ghetti, A., Tritto, T., Perez-Otano, I., Radcliffe, R.A., Seamans, J., Sejnowski, T., et al. 2001. The role of RNA editing of kainate receptors in synaptic plasticity and seizures. Neuron 29: 217-227.

Wang, Q., Khillan, J., Gadue, P., and Nishikura, K. 2000. Requirement of the RNA editing deaminase ADAR1 gene for embryonic erythropoiesis. Science 290: 1765-1768.

Wu, Y.M., Kung, S.S., Chen, J., and Chow, W.Y. 1996. Molecular analysis of cDNA molecules encoding glutamate receptor subunits, fGluR1 $\alpha$ and fGluR1 $\beta$, of Oreochromis sp. DNA Cell Biol. 15: 717725.

Zuker, M. 2003. Mfold web server for nucleic acid folding and hybridization prediction. Nucleic Acids Res. 31: 3406-3415. doi: 10.1093/nar/gkg595. 

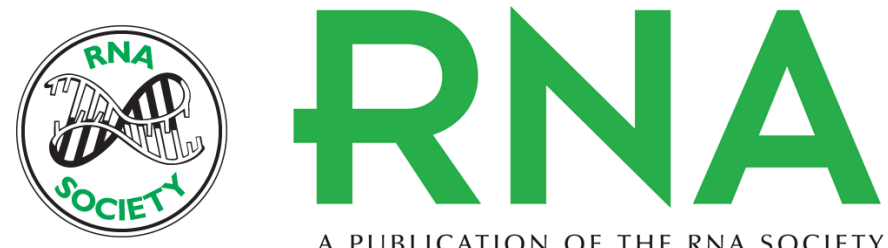

A PUBLICATION OF THE RNA SOCIETY

\section{A-to-I editing sites are a genomically encoded G: Implications for the evolutionary significance and identification of novel editing sites}

Nan Tian, Xiaojie Wu, Yaozhou Zhang, et al.

RNA 2008 14: 211-216

References This article cites 30 articles, 11 of which can be accessed free at:

http://rnajournal.cshlp.org/content/14/2/211.full.html\#ref-list-1

License

Email Alerting Receive free email alerts when new articles cite this article - sign up in the box at the Service top right corner of the article or click here. 